\title{
Distinct findings in primary Sjögren syndrome with positive anti-centromere antibodies
}

\author{
Claudia Cobilinschi ${ }^{1,2}$, Ioana Saulescu ${ }^{1,2}$, Cosmin Constantinescu ${ }^{1,2}$, \\ Constantin-Ioan Busuioc ${ }^{3}$, Ruxandra Ionescu ${ }^{1,2}$ \\ ${ }^{1}$ Department of Rheumatology and Internal Medicine, "Sf. Maria" Clinical Hospital, Bucharest, Romania \\ 2"Carol Davila" University of Medicine and Pharmacy, Bucharest, Romania \\ ${ }^{3}$ Histopathology Laboratory, "Sf. Maria" Clinical Hospital, Bucharest, Romania
}

\begin{abstract}
Primary Sjögren's syndrome (pSS) includes a rarer subtype with positive anti-centromere antibodies, which appears to be linked with scleroderma features and at higher risk of developing lymphoma.

Two female patients were evaluated in our department for severe dryness of the mouth and eyes. The younger patient had bilateral massive enlargement of her parotid glands, while the second had a history of recurrent corneal ulcers. They both described Raynaud's phenomenon and either had sclerodactily or facial teleangiectasias. The patients shared lymphopenia, negative anti-Ro and anti-La antibodies, positive high titer anti-centromere antibodies (ACA). The capillaroscopy exam revealed a scleroderma-like pattern. Minor gland biopsy reported a massive inflammatory lymphoplasmacytic infiltrate. One of the patients developed pulmonary lymphoma.

The ACA positive pSS is associated with more severe sicca symptoms but can also fulfill criteria for systemic sclerosis. More research is necessary to link this subtype to the risk of developing hematological malignancies.

Keywords: primary Sjögren syndrome, anticentromere antibodies, lymphoma, scleroderma features
\end{abstract}

\section{INTRODUCTION}

Sjögren's syndrome (SS) is a chronic autoimmune condition that typically targets exocrine glands through lymphocytic infiltrates, leading to a decrease in secretions and further glandular destruction (1). In theory, its relationship to other autoimmune diseases divides SS into primary (pSS) or secondary (sSS), the latter appearing in the presence of a clear diagnosis of connective tissue disease. However, 2012 ACR Criteria for Sjögren's state that distinguishing between primary and secondary is not entirely useful, since autoimmune pathogenesis is still unclear and the order of appearance of diseases is uncertain (2). Latest ACR-EULAR classification criteria published in 2016 are developed individually for pSS (3). Serologically, SS is usually associated with anti-Ro/SS-A or anti-La/SS-b antibodies. Primary SS includes a rarer subtype with positive anti-centromere antibodies (ACA) reported in 1.4$10.8 \%$ of patients (4). The presence of this immunological feature is associated with prominent sicca symptoms (xerostomia, xerophtalmia) intricated with mild to moderate limited scleroderma symptoms like sclerodactily and Raynaud's (5). Patients in this category might be at a higher risk of developing lymphoma (6).

We present two patients diagnosed with ACA positive pSS, their clinical and immunological features as well as disease outcome.

\section{CASE REPORT}

Demographical, clinical and laboratory data was collected from medical files in „Sf. Maria“ Clinical Hospital for two pSS patients with ACA.

Two female patients of 52 and 74 years old were evaluated in our department for severe dryness of the mouth and eyes.

The younger patient stated as previous medical history a chronic gastritis and a gastro-esophageal reflux. The patient had bilateral massive, painful swelling of her parotid glands (figure 1) with severe dryness of the mouth, multiple cavities, dysgeusia 


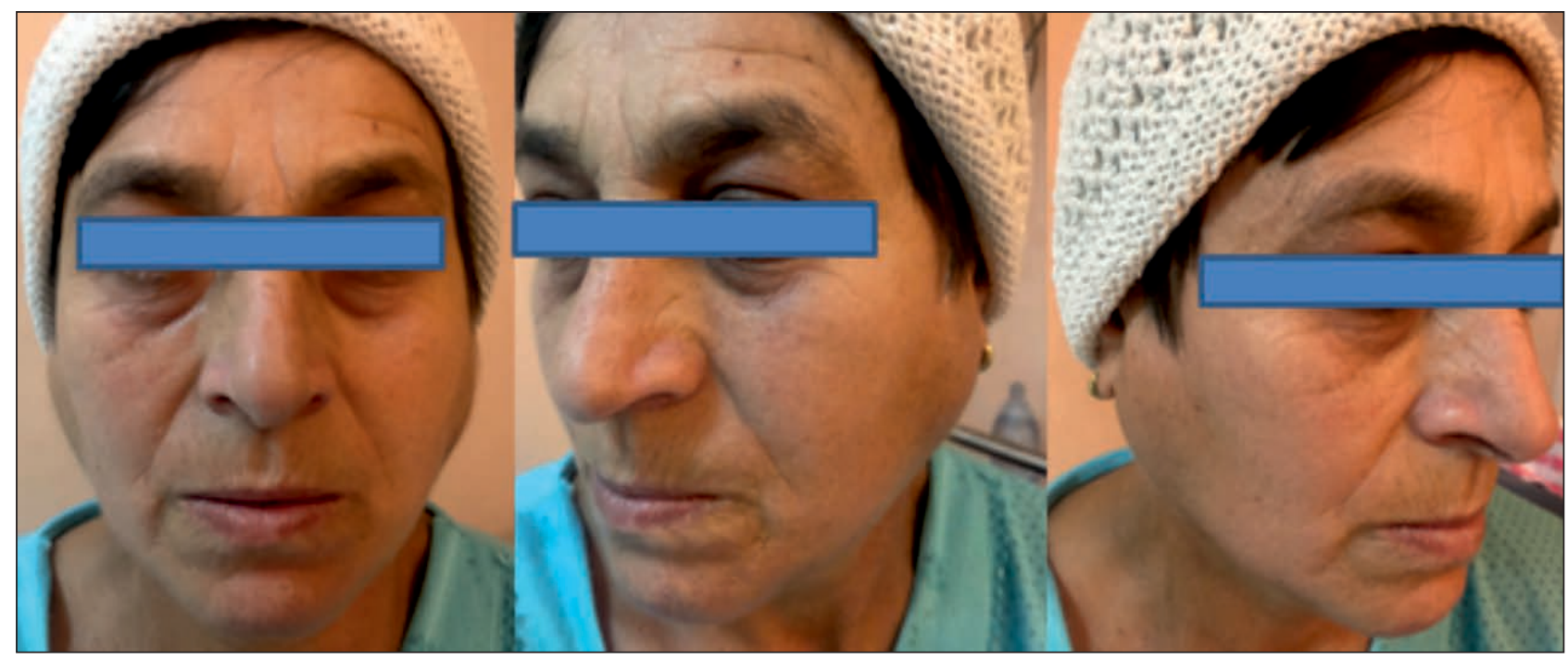

FIGURE 1. Bilateral parotid swelling in patient with $p S S$

and difficulties when speaking caused by lack of humectation. The clinical examination showed an intensely depapillated, erythematous tongue and angular cheilitis (figure 2).

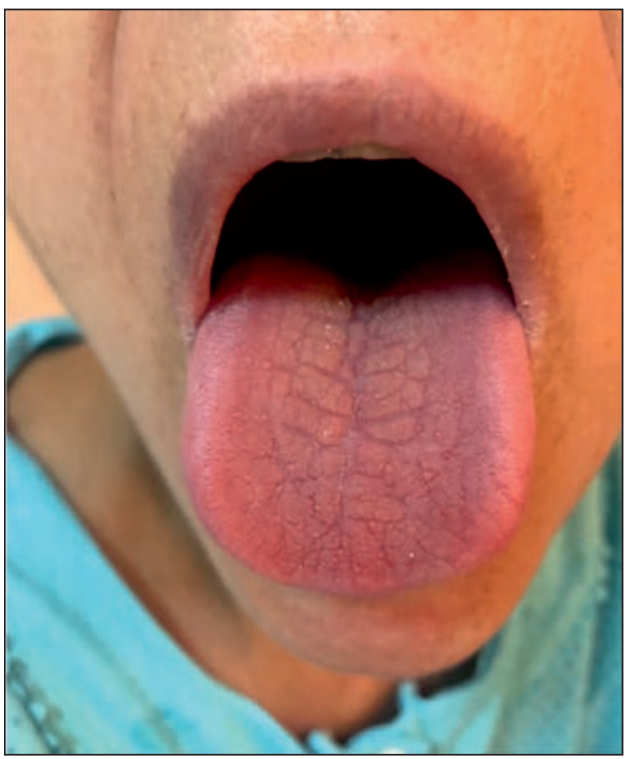

FIGURE 2. Erythematous tongue, angular cheilitis

Patient also complained of xerophtalmia and itchiness around the eyes. Figure 3 shows red, dry eyes. The ophthalmologist confirmed sicca conjunctivitis and positive Schirmer's test.

Dryness symptoms in first patient occurred more than three years ago and were accompanied by Raynaud's phenomenon for which she never referred to her general practitioner.

Due to her Raynaud's and associated sclerodactily we performed a nailfold capillaroscopy that revealed a reduced capillary density, megacapillaries and hemorrhages, suggestive of an active scleroderma pattern.

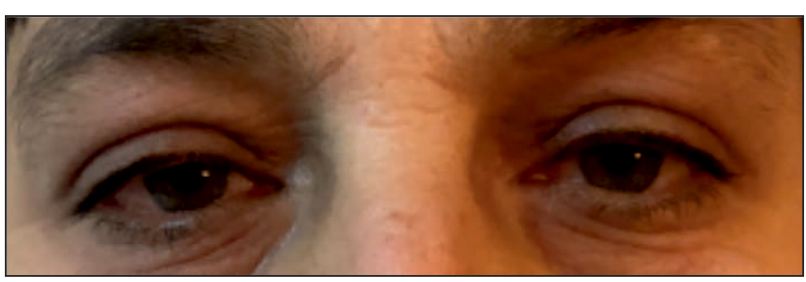

FIGURE 3. Bilateral reddish aspect of the eyes

Salivary gland ultrasound showed multiple cysts in both parotid and submandibular glands, surrounded by hyperechoic septa and hypoechoic formations over $6 \mathrm{~mm}$ according to Salaffi classification. Besides, the presence of hyperechoic formation is noted, probably sialolithiasis. The sonoelastography highlighted high stiffness areas overlaying the septa while the cysts and parenchymal degeneration correspond to "softer" areas (figure 4).

Serological investigations in this patient showed lymphopenia, low complement levels and high titer rheumatoid factor. She had 5 fold positive anti-nuclear antibodies, negative anti-Ro, anti-La, anti-U1RNP and anti-SCL70 antibodies, positive high titer anti-centromere antibodies (25 times higher upper reference value).

The minor salivary gland biopsy exposed a marked lymphoid infiltrate both intra and inter-lobules, a small area of macrophages and rare foreign body multinucleated cells, suggestive of a mucinous granuloma. Since an associated lymphoproliferation could not be excluded, further tests were made. Both the immunohistochemistry and molecular biology 


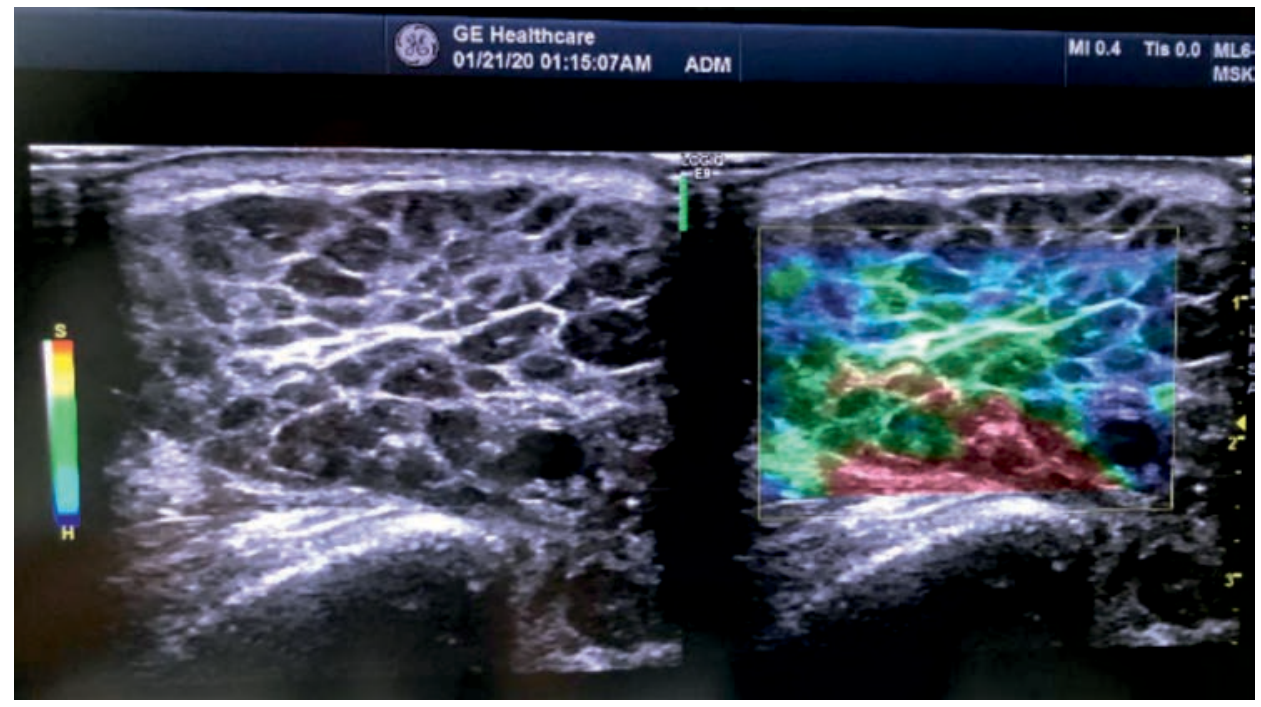

FIGURE 4. Parotid ultrasound and sonoelastography (hard - blue areas, soft - red and yellow areas)

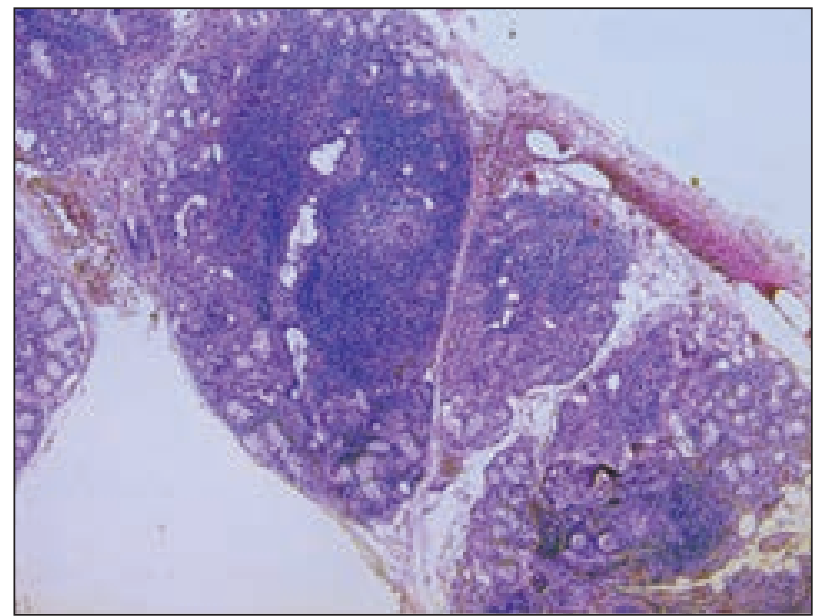

FIGURE 5. Tissue biopsy shows labial salivary glands infiltrated by multiple lymphocytic foci (each consisting of $>50$ lymphocytes), $5 x$

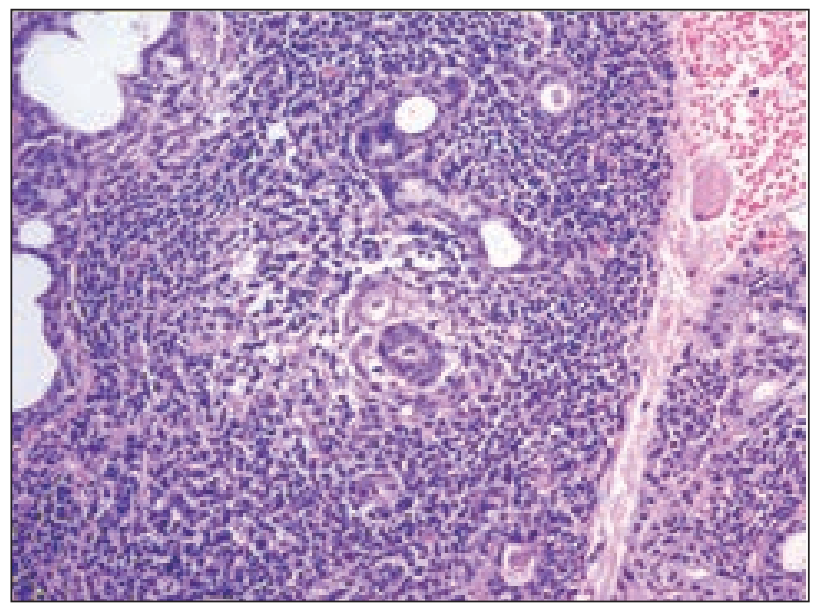

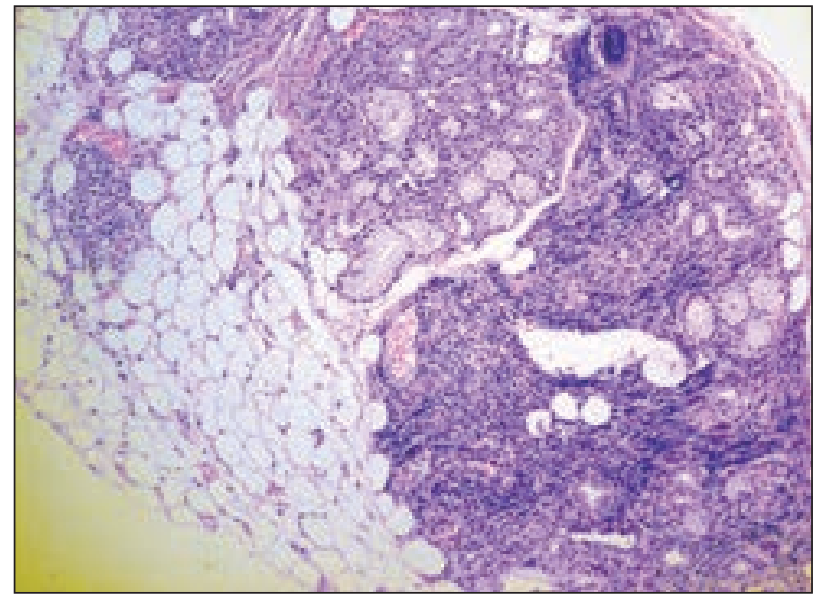

FIGURE 6. Uninvolved labial salivary gland (upper center) in contrast with focal lymphocytic sialadenitis (lower-right), 5x2

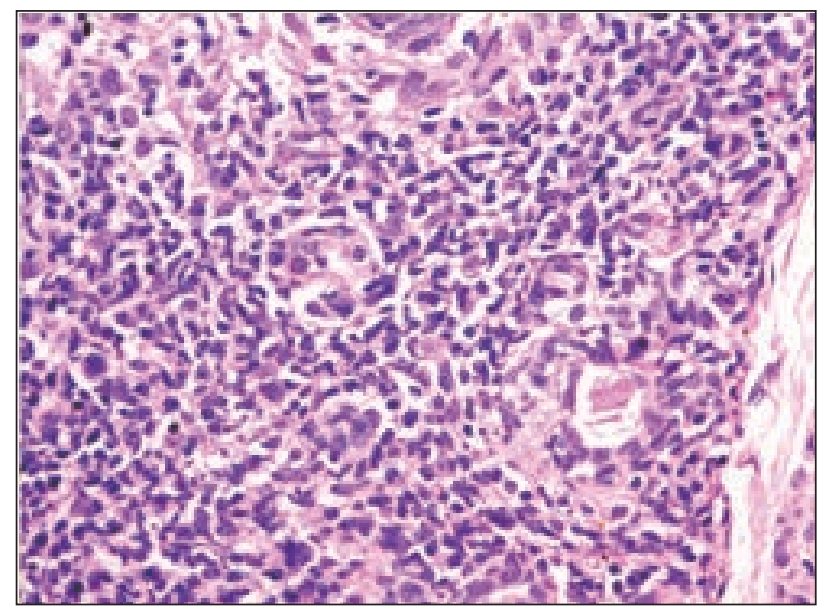

FIGURE 7. The infiltrate is dense, consisting mostly of lymphocytes, with scattered neutrophils and plasma cells and shows focal lymphoepithelial lesions (20x, 40x). PCR showed no clonality, excluding a MALT lymphoma

investigations concluded the lymphocytic infiltrate was solely reactive and had no malignant features.
The second patient, a 74-year-old female patient, had a prolonged history of recurrent corneal ulcers 
that she treated with current ophthalmologist. Later on she developed xerostomia and she also described Raynaud's phenomenon. The physical examination showed facial teleangiectasias, oral folds, angular cheilitis and cervical and submandibular lymph node enlargement. Barium swallowing test confirmed a gastroesophageal reflux.

As the previous patient, she shared the lymphopenia, a low $\mathrm{C} 4$, negative anti-Ro and anti-La antibodies and positive high titer anti-centromere antibodies. No changes in immunoglobulin levels were noted.

The capillaroscopy exam revealed a borderline pattern, with no typical changes for systemic sclerosis, while the gland ultrasound confirmed multiple hypoechoic structures of 2-3 $\mathrm{mm}$.

Minor gland biopsy reported a massive inflammatory lymphoplasmocytic infiltrate with a focus score of 3, indicating the diagnosis of SS.

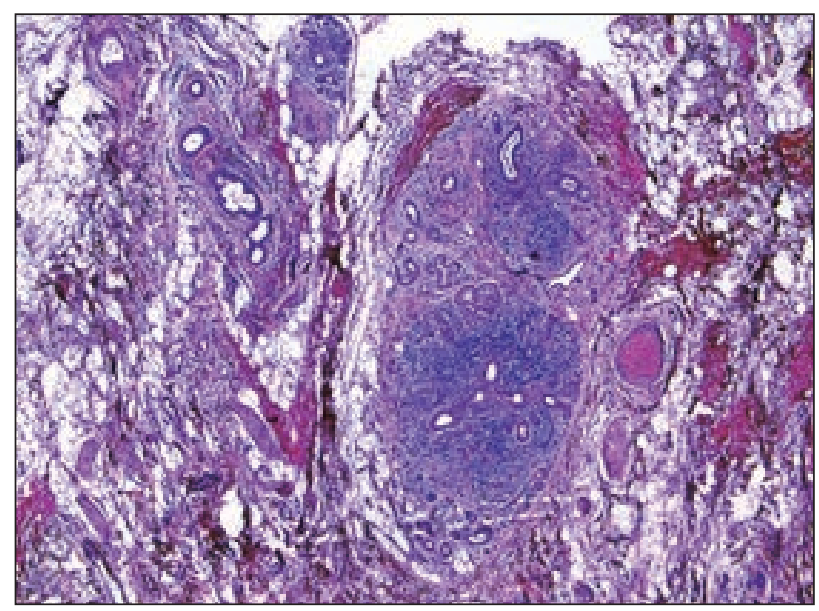

FIGURE 8. Labial salivary glands infiltrated by multiple lymphocytic foci. There is also peripheral fibrosis, congestion and interstitial hemorrhage, $5 x$

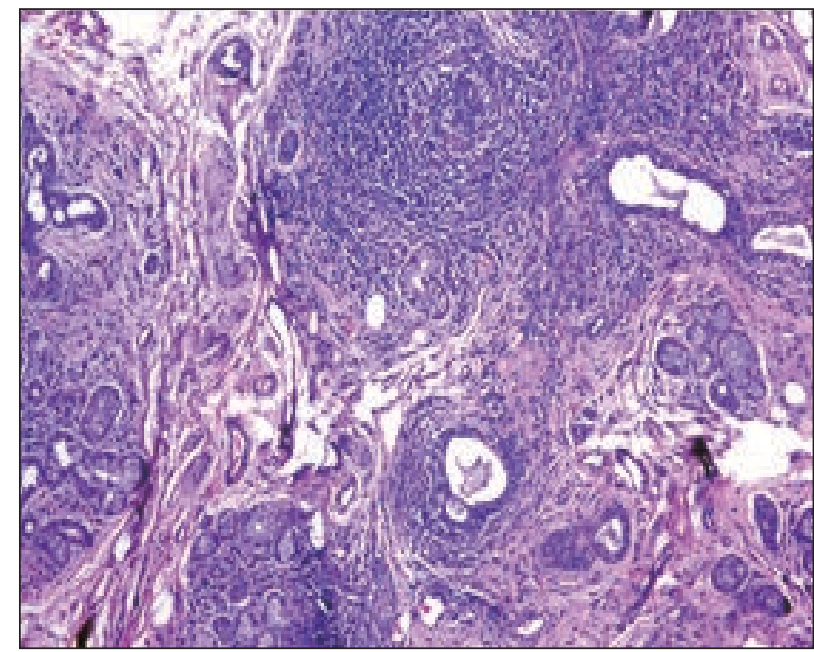

No patient was identified with pulmonary hypertension.

TABLE 1. Patient characteristics

\begin{tabular}{|c|c|c|}
\hline & Case 1 & Case 2 \\
\hline \multicolumn{3}{|l|}{ Demographics } \\
\hline- Age & 52 & 74 \\
\hline - Race & \multicolumn{2}{|l|}{ Caucasian } \\
\hline - Family history AID & \multicolumn{2}{|l|}{ No } \\
\hline \multicolumn{3}{|l|}{ Clinical features } \\
\hline - Ocular symptoms & Yes & $\begin{array}{c}\text { Prominent } \\
\text { (corneal ulcers) }\end{array}$ \\
\hline - Oral symptoms & $\begin{array}{l}\text { Prominent (no } \\
\text { taste, minimal } \\
\text { salivary flow) }\end{array}$ & Yes \\
\hline - Parotid enlargement & Yes & No \\
\hline - Raynaud's & Yes & \\
\hline - Lymphadenopathy & No & Yes \\
\hline - Other AID features & Sclerodactily & $\begin{array}{c}\text { Facial } \\
\text { teleangiectasias }\end{array}$ \\
\hline $\begin{array}{l}\text { - Extraglandular } \\
\text { involvement }\end{array}$ & \multicolumn{2}{|c|}{ Gastro-esophageal reflux } \\
\hline \multirow[t]{2}{*}{ Serology } & \multicolumn{2}{|c|}{$\begin{array}{l}\text { ANA ++ } \\
\text { Anti-centromere +++ } \\
\text { Lymphopenia } \\
\text { Negative anti-Ro,La }\end{array}$} \\
\hline & $\begin{array}{l}\text { Low complement } \\
\text { levels }(C 3, C 4) \\
\text { High titer RF }\end{array}$ & Low C4 \\
\hline Nailfold capillaroscopy & $\begin{array}{l}\text { Scleroderma-like } \\
\text { pattern }\end{array}$ & Borderline \\
\hline $\begin{array}{l}\text { Parotid ultrasound/ } \\
\text { imaging }\end{array}$ & \multicolumn{2}{|c|}{$\begin{array}{l}\text { Multiple hypoechoic formations } \\
\text { (cystic regions) }\end{array}$} \\
\hline $\begin{array}{l}\text { Minor salivary gland } \\
\text { biopsy }\end{array}$ & \multicolumn{2}{|c|}{$\begin{array}{l}\text { Massive lymphoplasmocitic } \\
\text { infiltrate, focus score }>1\end{array}$} \\
\hline
\end{tabular}

AID - autoimmune disease, ANA - anti-nuclear antibodies, RF - rheumatoid factor

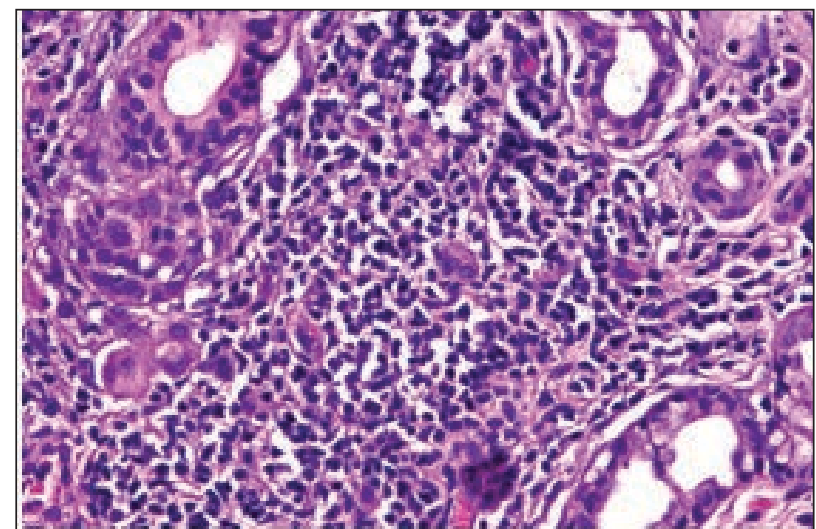

FIGURE 9. Dense lymphocytic infiltrate (each focus consisting of >50 lymphocytes) with benign lymphoepithelial lesions (10x, 40x) 


\section{CASE DISCUSSIONS}

Primary SS is characterized mainly by dry eyes, dry mouth due to glandular lymphocytic infiltration but it can also cause systemic damage. The majority of patients present with positive anti-Ro or anti-La antibodies, in accordance to the 2016 ACR-EULAR classification criteria for pSS (3). However, a SS subtype is considered in patients with positive anticentromere antibodies. ACA are not specific for systemic sclerosis, thus they can be identified in other autoimmune diseases such as systemic lupus erythematosus, primary biliary cirrhosis, mixed connective tissue disease of even rheumatoid arthritis, with variable prevalences (7).

Studies published so far indicate that ACA positive pSS have specific clinical and immunological features. Out of scleroderma manifestations, Raynaud's phenomenon seems to reach the highest prevalence in ACA-pSS patients, leading some authors to conclude it might be necessary to include this determination in SS associated with Raynaud's knowing that their presence can long precede clinical manifestations. Moreover, the severity of sicca symptoms was higher. The study from Ramos-Casals et al. analyzed clinical characteristics, and fulfillment of classification criteria for other systemic autoimmune diseases in 92 patients with pSS with negative anti-Ro/La (8). Out of the 8 ACA positives, three patients developed clinical features suggestive of limited scleroderma after a follow-up of six years; they had high titers of ANA and the development of incipient sclerodactily (8).

The study of Tsukamoto et al. published in 2018 aimed to clarify characteristics of ACA-positive SS patients. Out of the 585 patients diagnosed with pSS, 22 had ACA only, 464 had anti-SS-A antibodies only, 26 had both ACA and anti-SS-A antibodies, and 73 had neither ACA nor anti-SS-A antibodies (5). There was a higher proportion of patients with Raynaud's phenomenon or sclerodactyly in the ACA alone and double-positive groups. PSS patients with both ACA and anti-SS-A antibodies had Raynaud's phenomenon, sclerodactyly but also leukocytopenia and thrombocytopenia, similar to SS patients with anti-SS-A antibodies (5).

Pacients with SS have a known higher risk of developing lymphoma, the most frequent being the MALT non-Hodgkin histological subtype (9). Some of the unfavorable prognosis factors are persistent parotid gland enlargement, purpura, hypocomplementemia and the presence of cryoglobulins (10).

In 2010, two clinical case reports raised awareness of the possible link between the ACA-positive pSS subset and systemic manifestations or lymphoma. Both patients were initially classified as limited scleroderma but exhibited small vessel cutaneous vasculitis, parotid enlargement, low $\mathrm{C} 4$ complement levels, positive rheumatoid factor and were diagnosed with lymphoma (6).

Another study published in 2010 that described ACA-positive pSS characteristics notes that only two patients with systemic sclerosis out of the study group developed lymphoma and no ACA positive patients, making the risk not clinically significant (11).

Besides clinical distinctive manifestations that can occur in pSS-ACA positive, immunologic differences might be present. In systemic sclerosis, ACA target 3 centromeric proteins (CENP) identified as autoantigens: CENP-A, CENP-B, and CENP-C while in pSS the specific target of ACA is unknown (12).

In 2007, the study of Salliot et al. aimed to assess the significance of ACA in pSS by retrospectively diving patients in three groups - pSS with ACA without scleroderma, SS associated with another autoimmune disease, SS with ACA and limited scleroderma (7). Prevalence of ACA in this study was $4.7 \%$ and all recognized the same target CENP-B in the immunofluorescence test.

The presence of ACA can predict future development of pulmonary hypertension (13). However, in pSS with ACA positive subset, monitoring patients with regular echocardiography is an uncertain tool. A published abstract in 2019 questioned whether all patients identified with ACA should be screened for connective tissue diseases or whether they should undergo more detailed investigations for a potential gastro-esophageal reflux or pulmonary hypertension. Out of the total of 75 patients, 56 were referred to a Rheumatology Department and diagnosed with an autoimmune disease (14). Patients were tested with echocardiography and pulmonary function test and only one limited scleroderma patient was confirmed with pulmonary hypertension (14).

Our case reports confirm particular treats of pSS associated with ACA subtype that require careful diagnostic tools to differentiate from limited scleroderma and long-term follow-up. 


\section{CONCLUSIONS}

Primary SS with positive ACA have sicca symptoms that may be overlapping with systemic sclerosis manifestations but without fulfilling the 2013 ACR/EULAR classification criteria. Determining ACA is potentially valuable in establishing a defini-

\section{REFERENCES}

1. Ramos-Casals M, Brito-Zerón P, Sisó-Almirall A, Bosch X. Primary Sjogren syndrome. BMJ. 2012;344:e3821.

2. Shiboski SC, Shiboski CH, Criswell L, Baer A et al.; Sjögren's International Collaborative Clinical Alliance (SICCA) Research Groups. American College of Rheumatology classification criteria for Sjögren's syndrome: A data-driven, expert consensus approach in the Sjögren's International Collaborative Clinical Alliance cohort. Arthritis Care Res. (Hoboken). 2012;64(4):475-87.

3. Shiboski CH, Shiboski SC, Seror R, Criswell LA, Labetoulle $M$ et al.; International Sjögren's Syndrome Criteria Working Group. 2016 American College of Rheumatology/European League Against Rheumatism Classification Criteria for Primary Sjögren's Syndrome: A Consensus and Data-Driven Methodology Involving Three International Patient Cohorts. Arthritis Rheumatol. 2017;69(1):35-45.

4. Li Y, Bookman AAM. Anti-centromere antibody positive Sjögren's syndrome is associated with worse sicca symptoms than primary Sjögren's syndrome alone. J Rheumatol. 2019.

5. Tsukamoto M, Suzuki K, Takeuchi T. Clinical and Immunological Features of Anti-centromere Antibody-Positive Primary Sjögren's Syndrome. Rheumatol Ther. 2018:5:499-505.

6. Gulati D, Kushner I, File E, Magrey M. Primary Sjogren's syndrome with anticentromere antibodies - a clinically distinct subset. Clin Rheumatol. 2010;29(7):789-791.

7. Salliot C, Gottenberg JE, Bengoufa D, Desmoulins F, MiceliRichard C, Mariette X. Anticentromere antibodies identify patients tive diagnosis of this SS subtype and for medical management. The use of ACA for predicting pulmonary hypertension remains uncertain in pSS. More research is necessary to link this subtype to the risk of developing hematological malignancies.

\section{Conflict of interest: none declared} Financial support: none declared

with Sjögren's syndrome and autoimmune overlap syndrome. J Rheumatol. 2007;34(11):2253-2258.

8. Ramos-Casals M, Nardi N, Brito-Zerón P, et al. Atypical autoantibodies in patients with primary Sjögren syndrome: Clinical characteristics and follow-up of 82 cases. Semin Arthritis Rheum. 2006;35(5):312-321.

9. Alunno A, Leone MC, Giacomelli R, Gerli R, Carubbi F. Lymphoma and Lymphomagenesis in Primary Sjögren's Syndrome. Front Med (Lausanne). 2018:5:102.

10. Retamozo S, Brito-Zerón P, Ramos-Casals M. Prognostic markers of lymphoma development in primary Sjögren syndrome. Lupus. 2019;28(8):923-936

11. Bournia VK, Diamanti KD, Vlachoyiannopoulos PG, Moutsopoulos $\mathrm{HM}$. Anticentromere antibody positive Sjögren's syndrome: A retrospective descriptive analysis. Arthritis Res Ther. 2010;12(2):R47.

12. Song $\mathrm{G}$ et al. New centromere autoantigens identified in systemic sclerosis using centromere protein microarrays. J Rheumatol. 2013:40:461-468.

13. Kampolis C, Plastiras SC, Vlachoyiannopoulos PG, Moyssakis I, Tzelepis GE. The presence of anti-centromere antibodies may predict progression of estimated pulmonary arterial systolic pressure in systemic sclerosis. Scand J Rheumatol. 2008;37:278-283.

14. Alkoky H, Lazarus M. Should all patients with anti-centromere antibodies be referred for a rheumatology assessment? Ann Rheum Dis. 2019;78:1395. 